\title{
Dimension Synthesis of a Spatial Parallel Kinematic Manipulator Based on S-shaped Test Specimen
}

\author{
Yuzhe Liu, Jun Wu , Liping Wang and Jinsong Wang \\ State Key Laboratory of Tribology and Institute of Manufacturing Engineering, Department of Mechanical Engineering, Tsinghua University, \\ 100084 Beijing, China \\ Beijing Key Lab of Precision/Ultra-precision Manufacturing Equipments and Control, 100084 Beijing, China
}

\begin{abstract}
With the development of the aerospace industry, the hybrid machine tool for machining the complicated curved surface is becoming a research hotspot. The S-shaped test specimen proposed by Chengdu Aircraft Industrial Company is an effective test method to verify the ability of machining the complicated curved surface for a machine tool. Considering the spatial parallel kinematic manipulator in a hybrid machine tool provides the complicated orientation motion and its design of structure dimension affects the accuracy of the machine tool, this paper investigates the issue about dimension synthesis of the manipulator based on S-shaped test specimen. By establishing models of the S-shaped test specimen and parallel kinematic manipulator, the motion trajectory of the parallel kinematic manipulator for machining S-shaped test specimen is obtained. According to the motion trajectory and expected performance indices, the result of dimension synthesis for the manipulator is plotted and a set of suitable dimensions is achieved. With the analysis result in this paper, it is helpful to improving the accuracy of machining the S-shaped test specimen.
\end{abstract}

\section{Introduction}

With the rapid development of the aerospace industry, a 5 -axis machine tool with high accuracy, high speed and high orientational capability is becoming a research hotspot [1-4], which leads to two important issues: how to detect the machining performance of a 5-axis machine tool and which kind of machine tool is suitable.

For the first issue, many investigations have been addressed on it. In general, the accuracy detection test specimens are usually used as the detecting method. The most used one may be the ISO international standard test specimen, which usually is adopted to detect the translational axis accuracy of a 3-axis machine tool. However, this one cannot reflect the motion accuracy of multiple axes. Anther accuracy detection test specimen used a lot in practice is the NAS979 proposed by NASA in 1969. This one is used widely in 5-axis machine tools for detecting the motion accuracy of multiple axes. However, the most outstanding problem about this test specimen is that it cannot reflect the orientation accuracy of a 5-axis machine tool. Recently, in order to completely test the motion accuracy of multiple axes and satisfy the machining demand of the aerospace industry, a novel test specimen, named as the S-shaped test specimen and covering a mass of complicated machining poses, was proposed by Chengdu Aircraft Industrial Company, which has been applied successfully in a lot of factories and brought into ISO 10791-7 criterion group as an additional test specimen in the 78th ISO conference in
2015 [5-6]. Hence, it is necessary to investigate this new standard test specimen.

For the second issue, it is mainly to find a kind of 5axis machine tool which owns the performances of high accuracy, high speed and high orientational capability. 5axis machine tools usually can be divided into two categories: serial machine tools and serial-parallel (or hybrid) machine tools. It is well known that the serial machine tools are characterized by large workspace and simple kinematics but are subject to high inertia and error accumulation. The hybrid machine tool, combining the advantages of the parallel kinematic machines and the serial kinematic machines, is recently proposed and designed, which is more suitable for the aerospace industry [7-8]. In the past successful applications of 5axis hybrid machine tools, considering the structural features of serial and parallel mechanisms and the demands of the machining performance, a 3-Degree-OfFreedom (3-DOF) tool head with parallel kinematics and a 2-DOF translational table with serial kinematics are always adopted as the parallel module and the serial module in the 5-axis hybrid machine tool. Among them, the most successful application is the combination of a 3PRS parallel kinematic manipulator and a 2-DOF serial platform [9]. The 3PRS parallel kinematic manipulator is a spatial parallel kinematic manipulator and provides the orientation of the end effector of the 5-axis hybrid machine tool, which is the key component to guarantee the accuracy of machining aeronautical parts. 
Considering the special machining demand of the aerospace industry, such as the S-shaped test specimen covering a mass of complicated machining poses, and in order to improve the machining accuracy of this kind of 5 -axis hybrid machine tool which is used for the aerospace industry, it is necessary to study the relation between the 3PRS parallel kinematic manipulator and the S-shaped test specimen and deal with the dimension synthesis of the 3PRS parallel kinematic manipulator based on S-shaped test specimen. In order to achieve this goal, the most widely used method in the field of parallel kinematic manipulators, namely Performance-Chart based Design Methodology (PCbDM), is employed to investigate the dimension synthesis problem [10-11].

\section{Model of S-shaped test specimen}

The S-shaped test specimen consists of two similar ruled surfaces. For one ruled surface, it consists of a family of straight lines and is defined by two B-spline curves, which is shown in Fig.1. Its expression is written as:

$$
S(u, v)=(1-v) \times C_{1}(u)+v \times C_{2}(u)
$$

where $S(u, v)$ represents the function of the ruled surface. $C_{1}(u)$ represents the function of Curve 1. $C_{2}(u)$ represents the function of Curve 2. The parameters $u$ and $v$ are the variables of $S(u, v)$ and both vary in $[0,1]$.

One may see that the function of B-spline curve totally affects the shape of ruled surface. Hence, Chengdu Aircraft Industrial Company uses the De Boor-Cox method to completely define the function of B-spline curve in order to guarantee the uniqueness of the $\mathrm{S}$ shaped test specimen. The function of B-spline curve is expressed as:

$$
C(u)=\sum_{i=0}^{11} \boldsymbol{T}(u) \boldsymbol{M}_{i} \boldsymbol{P}_{i}
$$

where $C(u)$ represents the function of B-spline curve. $\boldsymbol{T}(u) \boldsymbol{M}_{i}$ defines the base function. $\boldsymbol{P}_{i}$ is the control points.

$\boldsymbol{T}(u)$ determines the order of the base function. As $C(u)$ is a three-order uniform rational B-spline curve, it leads to:

$$
\boldsymbol{T}(u)=\left[\begin{array}{llll}
1 & u & u^{2} & u^{3}
\end{array}\right]
$$

$\boldsymbol{M}_{i}$ determines the parameters of the base function. According to the De Boor-Cox method, $\boldsymbol{M}_{i}$ is determined in Table 1. $\boldsymbol{P}_{i}$ is given by Chengdu Aircraft Industrial Company and ignored in this paper. According to the Eqs (1) and (2), the model of S-shaped test specimen can be obtained and one of the ruled surfaces in S-shaped test specimen is plotted in Fig. 2. At this point, any orientations for machining the S-shaped test specimen can be obtained by the model.

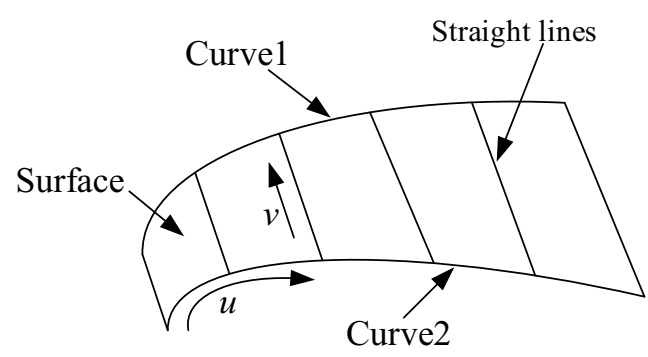

Figure 1. The ruled surface diagram

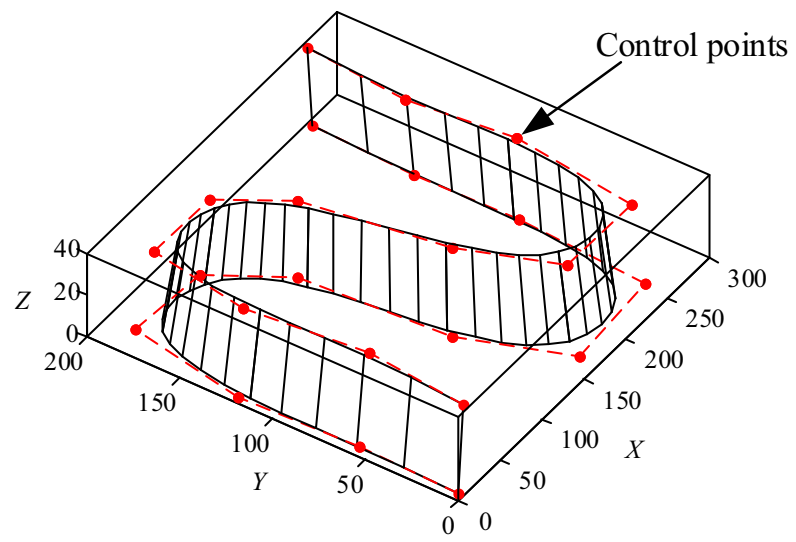

Figure 2. One of the ruled surface in S-shaped test specimen

Table 1. Definition of $\boldsymbol{M}_{i}$

\begin{tabular}{|c|cc|}
\hline$i$ & $\boldsymbol{M}_{i}$ \\
\hline \multirow{4}{*}{0} & {$\left[\begin{array}{cccc}1 & 0 & 0 & 0 \\
-3 & 3 & 0 & 0 \\
3 & -9 / 2 & 3 / 2 & 0 \\
-1 & 7 / 4 & -11 / 12 & 1 / 6\end{array}\right]$} \\
\hline \multirow{4}{*}{1} & {$\left[\begin{array}{cccc}1 / 4 & 7 / 12 & 1 / 6 & 0 \\
-3 / 4 & 1 / 4 & 1 / 2 & 0 \\
3 / 4 & -5 / 4 & 1 / 2 & 0 \\
-1 / 4 & 7 / 12 & -1 / 2 & 1 / 6\end{array}\right]$} \\
\hline \multirow{4}{*}{$2 \sim 6$} & {$\left[\begin{array}{cccc}1 / 6 & 2 / 3 & 1 / 6 & 0 \\
-1 / 2 & 0 & 1 / 2 & 0 \\
1 / 2 & -1 & 1 / 2 & 0 \\
-1 / 6 & 1 / 2 & -1 / 2 & 1 / 6\end{array}\right]$} \\
\hline \multirow{4}{*}{7} & {$\left[\begin{array}{cccc}1 / 6 & 2 / 3 & 1 / 6 & 0 \\
-1 / 2 & 0 & 1 / 2 & 0 \\
1 / 2 & -1 & 1 / 2 & 0 \\
-1 / 6 & 1 / 2 & -7 / 12 & 1 / 4\end{array}\right]$} \\
\hline \multirow{4}{*}{8} & {$\left[\begin{array}{cccc}1 / 6 & 7 / 12 & 1 / 4 & 0 \\
-1 / 2 & -1 / 4 & 3 / 4 & 0 \\
1 / 2 & -5 / 4 & 3 / 4 & 0 \\
-1 / 6 & 11 / 12 & -7 / 4 & 1\end{array}\right]$} \\
\hline
\end{tabular}

\section{Error model of the 3PRS parallel kinematic manipulator}

The 3PRS parallel kinematic manipulator is shown in Fig. 3. It is composed of a moving platform, a base platform and three supporting legs with identical kinematic structure. Each PRS leg contains one P joint, one R joint and one $\mathrm{S}$ joint. A fixed Cartesian reference coordinate system $O-X Y Z$ is fixed at the center of the base platform 
$B_{1} B_{2} B_{3}$. A moving Cartesian reference coordinate system $N-U V W$ is fixed at the center of the moving platform $A_{1} A_{2} A_{3}$. For simplicity and without losing the generality, let the $X$-axis point along the direction of vector $O B_{1}$ and the $U$-axis point along the vector $O A_{1} . B_{i} C_{i}$ on the base platform for $i=1,2$ and 3 represents the guide rail of each leg. The guide rail of each leg is perpendicular to the base platform. Each $\mathrm{P}$ joint moves along $B_{i} C_{i}$. $\mathrm{P}$ joint connects the base platform to $\mathrm{R}$ joint. $\mathrm{R}$ joint connects the $\mathrm{P}$ joint to the $C_{i} A_{i}$ link. $\mathrm{S}$ joint (attached to the moving platform) connects the moving platform to $C_{i} A_{i}$ link. Furthermore, all the joints attached to the base and moving platform are symmetrically distributed at vertices of the equilateral triangles.

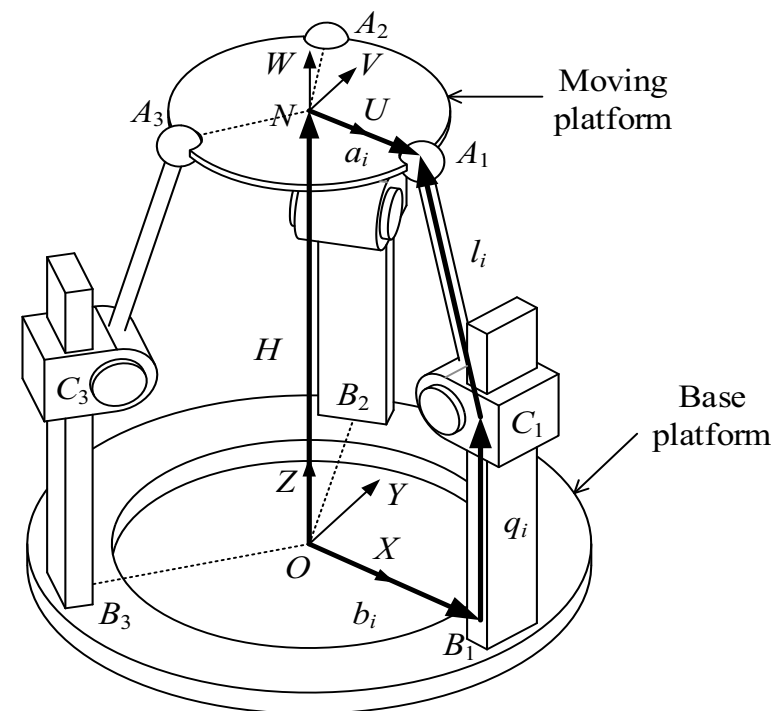

Figure 3. Structure of 3PRS parallel kinematic manipulator

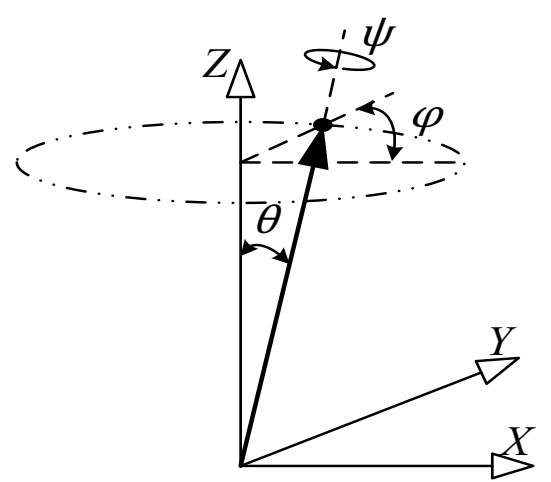

Figure 4. TT angle

In order to simplify the orientation expression, TT rotation angle in Fig. 4 is introduced to describe the orientation of the moving platform. The rotational matrix can be expressed as:

$$
\begin{aligned}
\boldsymbol{R}_{\mathrm{TT}}(\varphi, \theta, \psi)= & {\left[\begin{array}{ccc}
\cos \varphi & -\sin \varphi & 0 \\
\sin \varphi & \cos \varphi & 0 \\
0 & 0 & 1
\end{array}\right]\left[\begin{array}{ccc}
\cos \theta & 0 & \sin \theta \\
0 & 1 & 0 \\
-\sin \theta & 0 & \cos \theta \\
\cos \varphi & \sin \varphi & 0 \\
-\sin \varphi & \cos \varphi & 0 \\
0 & 0 & 1
\end{array}\right]\left[\begin{array}{ccc}
\cos \psi & -\sin \psi & 0 \\
\sin \psi & \cos \psi & 0 \\
0 & 0 & 1
\end{array}\right] }
\end{aligned}
$$

The angle $\psi$ rotates around the normal direction of the moving platform and it is zero in an ideal situation.
Furthermore, the accuracy of the angle $\psi$ is not necessary to guarantee when the machine tool machines a part. Hence, $\psi$ can be ignored and only two angles are kept in the rotational matrix.

Based on the vector loop $O B_{i} C_{i} A_{i} N$, the kinematic model can be written as:

$$
\boldsymbol{H}+\boldsymbol{R}_{\mathrm{TT}} \boldsymbol{R}_{i} \boldsymbol{a}_{i}=\boldsymbol{R}_{i} \boldsymbol{b}_{i}+\boldsymbol{R}_{i} \boldsymbol{R}_{B i} \boldsymbol{q}_{i}+\boldsymbol{R}_{i} \boldsymbol{R}_{B i} \boldsymbol{R}_{C i} \boldsymbol{l}_{i}
$$

where $\boldsymbol{R}_{i}$ represents the rotation matrix of each leg. Furthermore, $\boldsymbol{a}_{i}, \boldsymbol{b}_{i}$ and $\boldsymbol{l}_{i}$ represent the position vectors of $N A_{i}, O B_{i}$ and $C_{i} A_{i} . \boldsymbol{H}$ represents the position vector of the moving platform. $\boldsymbol{q}_{i}$ represents the input vector of each $\mathrm{P}$ joint. The orientation of the $\mathrm{P}$ joint and the orientation of the $\mathrm{R}$ joint are represented by matrices $\boldsymbol{R}_{\mathrm{B} i}$ and $\boldsymbol{R}_{\mathrm{C} i}$, respectively.

Based on the normal kinematic model above, the error model can be obtained by taking perturbations on both side of Eq. (4):

$$
\begin{aligned}
& \boldsymbol{H}+\Delta \boldsymbol{H}+(\boldsymbol{E}+\Delta \boldsymbol{\alpha} \times) \boldsymbol{R}_{\mathrm{TT}} \boldsymbol{R}_{i}\left(\boldsymbol{a}_{i}+\Delta \boldsymbol{a}_{i}\right) \\
& =\boldsymbol{R}_{i}\left(\boldsymbol{b}_{i}+\Delta \boldsymbol{b}_{i}\right)+\boldsymbol{R}_{i}\left(\boldsymbol{E}+\Delta \boldsymbol{\theta}_{B i} \times\right) \boldsymbol{R}_{B i} \boldsymbol{e}_{z i}\left(q_{i}+\Delta q_{i}\right) \\
& +\boldsymbol{R}_{i}\left(\boldsymbol{E}+\Delta \boldsymbol{\theta}_{B i} \times\right) \boldsymbol{R}_{B i}\left(\boldsymbol{E}+\Delta \boldsymbol{\theta}_{C i} \times\right) \boldsymbol{R}_{C i} \boldsymbol{\omega}_{i}\left(l_{i}+\Delta l_{i}\right)
\end{aligned}
$$

where the parameters with a triangular symbol represent the corresponding error parameters in the error model.

\section{Determining the parameter design space and evaluation indices}

\subsection{Determining the parameter design space}

In order to keep the isotropy of the 3PRS parallel kinematic manipulator, each of $\left|a_{i}\right|,\left|b_{i}\right|$ and $\left|l_{i}\right|$ is kept in the same so that there are 3 parameters to design it, namely $a, b$ and $l$. Let

$$
D=(a+b+l) / 3
$$

Then

$$
\begin{aligned}
& d_{1}=a / D \\
& d_{2}=b / D \\
& d_{3}=l / D
\end{aligned}
$$

Hence

$$
d_{1}+d_{2}+d_{3}=3
$$

According to the working mode of the manipulator, two geometric constraint equations of the parameters can be derived as:

$$
\begin{gathered}
d_{3} \geq d_{2}-d_{1} \\
d_{2} \geq d_{1}
\end{gathered}
$$




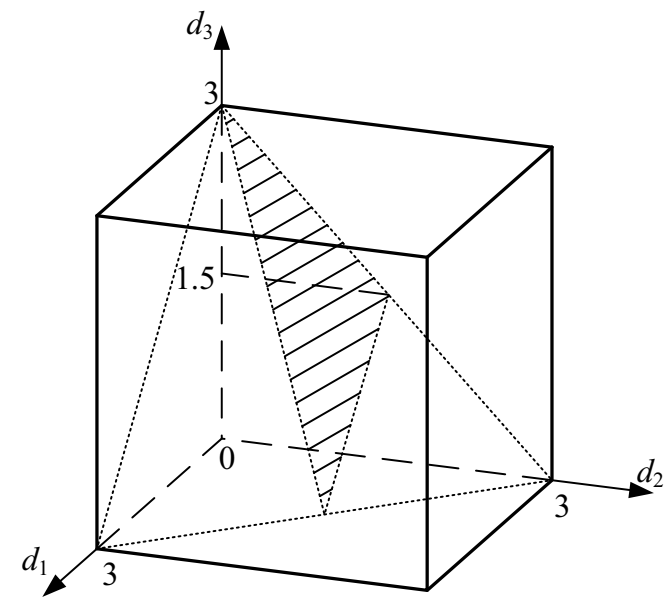

Figure 5. Parameter design space

Using Eqs. (10)-(12), a planar region can be obtain in Fig. 5, which is called the parameter design space. For the convenience of illustration, this region is transformed into a planar system defined by $s$ and $t$, as shown in Fig. 6 . The mapping function of the related variables can be expressed as:

$$
\begin{gathered}
t=d_{3} \\
s=\frac{2 d_{2}}{\sqrt{3}}+\frac{d_{3}}{\sqrt{3}}
\end{gathered}
$$

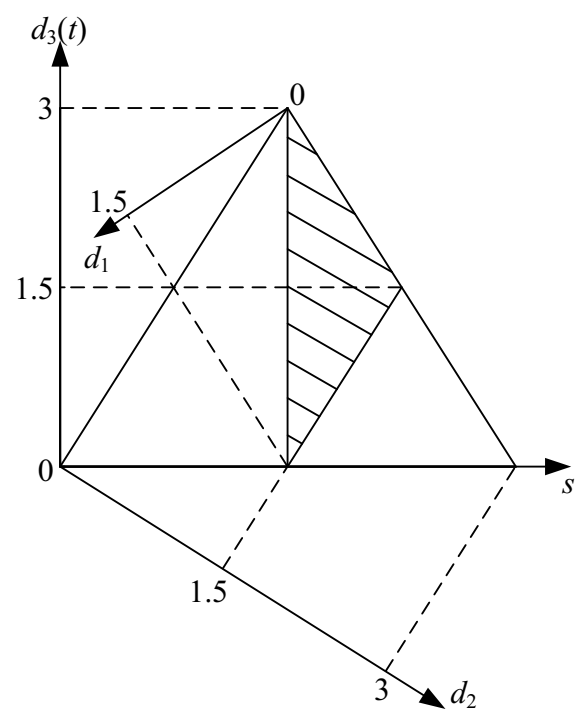

Figure 6. Planar design region

\subsection{Evaluation indices}

In order to improve the machining accuracy of this kind of 5-axis hybrid machine tool which is used for the aerospace industry, the workspace, position and orientation error sensitivity based on the machining trajectory of S-shaped test specimen are taken as the performance indices of concern to investigate.

For the same input range (assumed as $[-q, q]$ for each leg) and considering the isotropy of the manipulator, the performance index of workspace can be expressed as:

$$
w=\sin \{\theta[\text { forward_solution }(\boldsymbol{q})]\}
$$

where this index $w$ represents the sine function value of $\theta$ solved by forward solution based on the input $q=[q, q,-q]$.

A determination of a global position error sensitivity based on the machining trajectory of S-shaped test specimen is put forward in the following:

$\sigma_{\mathrm{H} m}=\frac{\int_{V} \sqrt{\left[\frac{\partial\left(\Delta H_{\mathrm{x}}\right)}{\partial\left(\Delta P_{\mathrm{H} m}\right)}\right]^{2}+\left[\frac{\partial\left(\Delta H_{\mathrm{y}}\right)}{\partial\left(\Delta P_{\mathrm{H} m}\right)}\right]^{2}+\left[\frac{\partial\left(\Delta H_{\mathrm{z}}\right)}{\partial\left(\Delta P_{\mathrm{H} m}\right)}\right]^{2}} d V}{V}$

where $V$ denotes the machining trajectory of S-shaped test specimen. $\Delta H_{\mathrm{x}}, \Delta H_{\mathrm{y}}$ and $\Delta H_{\mathrm{z}}$ respectively represent elements of the position error vector of the end effector. $\Delta P_{\mathrm{H}}$ represents an element of the position error vector of a joint. $m$ is the number of position error parameters of joints.

Then

$$
\sigma_{\mathrm{H}}=\max \left(\sigma_{\mathrm{H} m}\right)
$$

where $\sigma_{\mathrm{H}}$ is the maximum $\sigma_{\mathrm{H} m}$, namely the index of position error sensitivity.

Similarly, a determination of a global orientation error sensitivity based on the machining trajectory of S-shaped test specimen is put forward in the following:

$\sigma_{\alpha m}=\frac{\int_{V} \sqrt{\left[\frac{\partial\left(\Delta \alpha_{\mathrm{x}}\right)}{\partial\left(\Delta P_{\alpha m}\right)}\right]^{2}+\left[\frac{\partial\left(\Delta \alpha_{\mathrm{y}}\right)}{\partial\left(\Delta P_{\alpha m}\right)}\right]^{2}+\left[\frac{\partial\left(\Delta \alpha_{\mathrm{z}}\right)}{\partial\left(\Delta P_{\alpha m}\right)}\right]^{2}} d V}{V}$

$$
\sigma_{\alpha}=\max \left(\sigma_{\alpha m}\right)
$$

where $\sigma_{\alpha}$ is the index of orientation error sensitivity.

It should be noted that the above trajectory based on S-shaped test specimen just denotes the orientation trajectory because the orientation error is the main error source and the position error is easy to guarantee in the machine tool.

\section{Dimension synthesis}

Before dimension synthesis, the ranges of evaluation indices should be determined. Considering the small error sensitivities are helpful for improving the accuracy and the large workspace is helpful for satisfying the machining demand, the ranges of evaluation indices are determined as: $w>0.8, \sigma_{\mathrm{H}}<0.72$ and $\sigma_{\alpha}<20$. According to the value requirements, the result of dimension synthesis is plotted with the shadow area in Fig. 7. The dimension satisfied with the evaluation indices has been covered by the shadow area. A solution can be selected and the corresponding dimensions can be obtained. In this paper, it is assumed that $D=302 \mathrm{~mm}$ and the selected point is $(s$, $t)=(1.92,1.5)$. Then, $a=177 \mathrm{~mm}, b=276 \mathrm{~mm}$ and $l=453$ mm. 


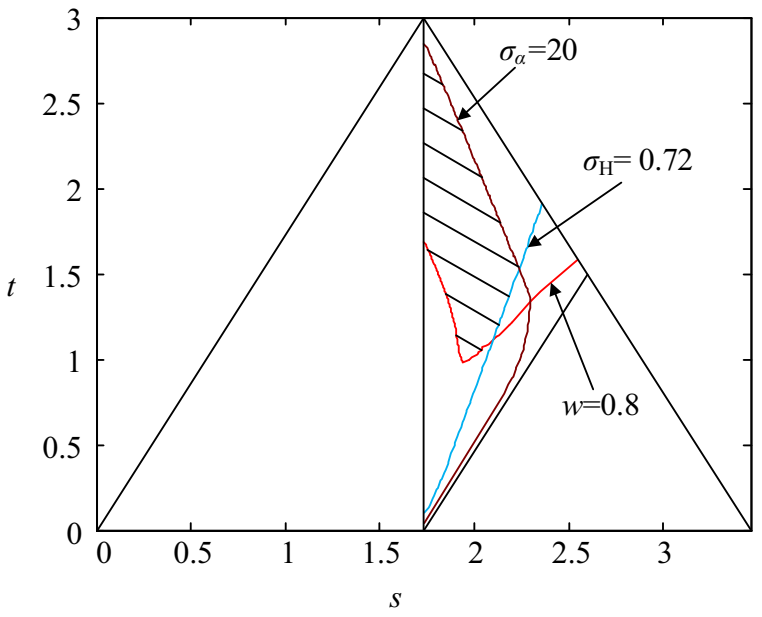

Figure 7. Optimum region for the manipulator

\section{Conclusions}

Based on the machining trajectory of S-shaped test specimen, this paper investigates the dimension synthesis of the 3PRS parallel kinematic manipulator used in a hybrid machine tool. By analyzing the mathematical characteristics of a ruled surface and using the data provided by Chengdu Aircraft Industrial Company, the model of S-shaped test specimen is established and the corresponding orientations for machining the S-shaped test specimen are obtained. In order to investigate the relation between the machining trajectory accuracy of Sshaped test specimen and the 3PRS parallel kinematic manipulator, the kinematic model and error model of the manipulator are established, respectively. According to the parameter design space determined by the working mode of the manipulator and the expected performance indices, the result of dimension synthesis is plotted. Within the dimension synthesis solutions, a set of suitable dimensions of the 3PRS parallel kinematic manipulator, namely $a=177 \mathrm{~mm}, b=276 \mathrm{~mm}$ and $l=453 \mathrm{~mm}$, is achieved. With this set of dimensions, it is helpful to improving the accuracy of machining the S-shaped test specimen.

\section{Acknowledgements}

This work is supported by the National Natural Science Foundation of China (Grant No. 51575307 and 51225503), the Science and Technology Major ProjectAdvanced NC Machine Tools \& Basic Manufacturing Equipments (2013ZX04004021 and 2014ZX04002051), and Top-Notch Young Talents Program of China.

\section{References}

1. J. Wu, J. Wang, L. Wang, T. Li. Dynamics and control of a planar 3-DOF parallel manipulator with actuation redundancy. Mech. Mach. Theory 44(4), 835-849 (2009)

2. R. Ramesh, M.A. Mannan, A.N. Poo. Error compensation in machine tools - a review: Part I: geometric, cutting-force induced and fixturedependent errors. Int. J. Mach. Tools Manuf. 40(9), 1235-1256 (2000)

3. P.S. Rao, N.M. Rao. Position analysis of spatial 3RPS parallel manipulator. Int. J. Mech. Eng. Rob. Res. 2(2), 80-90 (2013)

4. V.V. Kamesh, A.B.S. Rao, K.M. Rao. A computer based technique for structural comparison of in parallel robotic manipulators applicable for higher pairs. Int. J. Mech. Eng. Rob. Res. 1(1), 107-119 (2012)

5. Z. Su, L. Wang. Latest development of a new standard for the testing of five-axis machine tools using an S-shaped test piece. Proc. Inst. Mech. Eng. Part B-J. Eng. Manuf. 229(7), 1221-1228 (2015)

6. Z. Jiang, J. Ding, Z. Song, L. Du, W. Wang. Modeling and simulation of surface morphology abnormality of ' $\mathrm{S}$ 'test piece machined by five-axis CNC machine tool. Int. J. Adv. Manuf. Technol. 1$15(2015)$

7. F. Xie, X.J. Liu, T. Li. A comparison study on the orientation capability and parasitic motions of two novel articulated tool heads with parallel kinematics. Adv. Mech. Eng. 5, 249103 (2013)

8. T. Huang, M. Li, X.M. Zhao, J.P. Mei, D. G. Chetwynd, S.J. Hu. Conceptual design and dimensional synthesis for a 3-DOF module of the TriVariant-a novel 5-DOF reconfigurable hybrid robot. IEEE Trans. Robot. 21(3), 449-456 (2005)

9. P. Huang, J. Wang, L. Wang, R. Yao. Kinematical calibration of a hybrid machine tool with Regularization method. Int. J. Mach. Tools Manuf. 51(3), 210-220 (2011)

10. X.J. Liu, J. Wang. A new methodology for optimal kinematic design of parallel mechanisms. Mech. Mach. Theory 42(9), 1210-1224 (2007)

11. F. Xie, X.J. Liu, J. Wang. A 3-DOF parallel manufacturing module and its kinematic optimization. Robot. Comput.-Integr. Manuf. 28(3), 334-343 (2012) 